\title{
IMPRESIÓN Y ADAPTACIÓN. La construcción de la identidad del área central de la ciudad de Córdoba
}

\author{
Mariana Debat \\ Universidad Nacional de Córdoba. \\ Programa de Doctorado Universitat Politècnica de Catalunya
}

\section{RESUMEN}

Este artículo indaga sobre la construcción del Área Central de la ciudad de Córdoba (Argentina). Frente a la idea ampliamente extendida de que las áreas centrales de las ciudades de fundación hispana en América Latina conforman un grupo homogéneo, defiendo la hipótesis de que cada una ha configurado una imagen única a partir de un mismo modelo de origen y sus variaciones posteriores.

\section{ABSTRACT}

This paper studies the construction of the Central Area of the city of Cordoba (Argentina). In front to the extended idea that the central areas of spanish founded cities in Latin America conform a homogenous group, it defends the hypothesis that each one is a singular and unique image based on the same model and its subsequent variations. 


\section{INTRODUCCIÓN}

Entre los estudios más tradicionales en el campo del urbanismo se ha extendido la idea de que tras el proyecto de fundación las ciudades latinoamericanas son producto de la improvisación y de la falta de reglas en su crecimiento. Se sostiene además, que no ha habido suficientes instrumentos de reflexión e incluso se duda de su existencia. Esos mismos estudios, generalmente marcados por una mirada eurocéntrica, argumentan que en su construcción se ha dado una transferencia directa de modelos de otras latitudes, sin tener en cuenta el contexto local o las pre-existencias. Sin embargo, en una investigación en curso de la que este artículo forma parte, negamos este argumento, sostenemos la existencia de reflexión y defendemos el papel que las ideas de ciudad han jugado en la definición de su identidad. ${ }^{1}$

Teniendo en cuenta este marco general nos proponemos explorar la identidad del área central de la ciudad de Córdoba, que al igual que la mayoría de las ciudades de fundación hispana en América Latina parte de un modelo geométrico. Éste ha llevado a la simplificación, en los estudios más extendidos, de englobar en un mismo grupo a todas las ciudades de origen español. En general se cree que las áreas centrales de estas ciudades son bastante similares. No obstante se puede demostrar que, aunque existe un denominador común que no se puede obviar, el proceso de desarrollo y las reflexiones que se sucedieron juegan un papel igual determinante en su configuración. Es decir, que en cada ciudad podemos descubrir una identidad propia, producto de las sinergias entre el modelo "impuesto," sus variaciones y adaptaciones y la superposición de reflexiones y proyectos que se construyen en el tiempo.

${ }^{1}$ La investigación a la que hacemos referencia se titula Entre la abstracción y la realidad. Ideas de ciudad en la construcción de Córdoba y Rosario, presentada como tesina de Máster en marzo de 2010 en el Departamento de Urbanismo y Ordenación del Territorio de la Universidad Politécnica de Catalunya. En ella se defiende la existencia de reflexión urbana en las ciudades latinoamericanas de segundo orden, como el caso de Córdoba. Pero además que las ideas de ciudad propuestas en dicha reflexión dejan su impronta en la ciudad, en la imagen y la estructura urbana contemporánea y que han jugado un papel relevante en la definición de su identidad. Para desarrollarlo identificamos seis episodios relevantes, entre los cuales la etapa fundacional, constituye el primero.

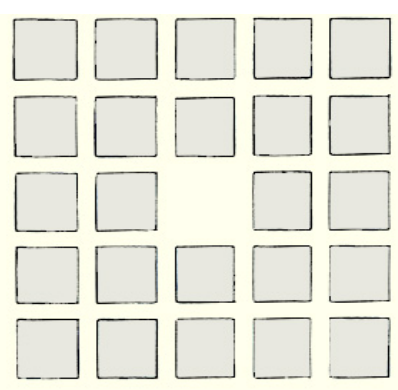

Modelo teórico

Constante, uniforme y genérico Fuente: El sueño de un orden

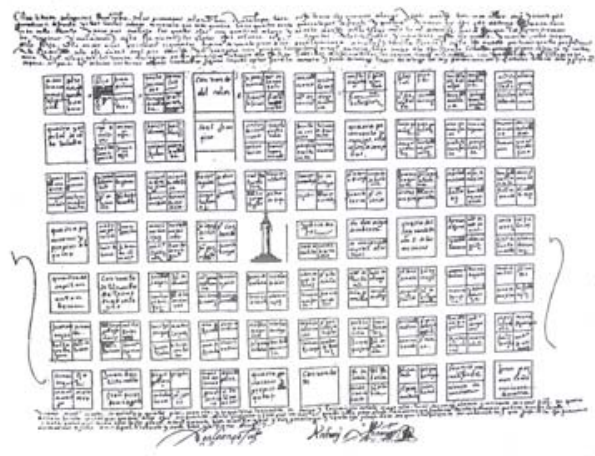

Primer grado de ajuste: el modelo toma sus propias medidas

Fuente: plano de Suarez de Fiqueroa de 1577 Archivo Municipal de Córdoba 


\section{IMPRESIÓN. EI modelo geométrico en el origen de la ciudad}

Es bien sabido que en su origen la mayoría de las ciudades españolas en Latinoamérica solo existen en un plano. La ciudad es un proyecto que debe construirse con el tiempo y cuyo trazado solo revela la ambición del grupo fundador. $^{2}$ Este largo proceso de transformación del territorio natural se lleva a cabo a través de la idea de impresión de un modelo geométrico artificial, que en principio se propone genérico y repetitivo, sobre un territorio que se concibe plano e indiferente a las pre-existencias. Estas características han llevado a la simplificación de englobar a todas las ciudades de origen español en un mismo grupo, cuando en realidad a pesar de partir de un modelo similar, difieren mucho unas de otras.

La estructura base del área central de Córdoba responde a lo que algunos autores denominan "tipo urbano consagrado", es decir el trazado en cuadrícula anterior a las leyes de Felipe II. ${ }^{3}$ En el primer plano conocido de la ciudad (Lorenzo Suarez de Figueroa de 1577) el modelo teórico adquiere un primer grado de ajuste a la realidad al determinar dimensiones concretas y definir su extensión.

El trazado base está constituido por un damero de calles rectilíneas que definen un total de 70 manzanas cuadradas de 440 pies de lado (122,6 m), prácticamente iguales, y calles de 35 pies $(10,67 \mathrm{~m})$. La subdivisión predominante de las manzanas es en cuatro lotes de $220 \times 220$ pies $(61,3 \mathrm{~m} \times$ $61,3 \mathrm{~m}){ }^{4}$ Se otorgan los solares a sus futuros dueños, constituyendo así también una transacción legal de adjudicación de tierras.

\footnotetext{
${ }^{2}$ Las ciudades más grandes: como Buenos Aires, Lima o México, sobrepasan las cien manzanas; mientras que la mayoría se encuentra sobre las veinticinco. Córdoba tiene en su origen setenta manzanas.

${ }^{3}$ AAVV. (1994)

${ }^{4}$ Óp. Cit.3
} 


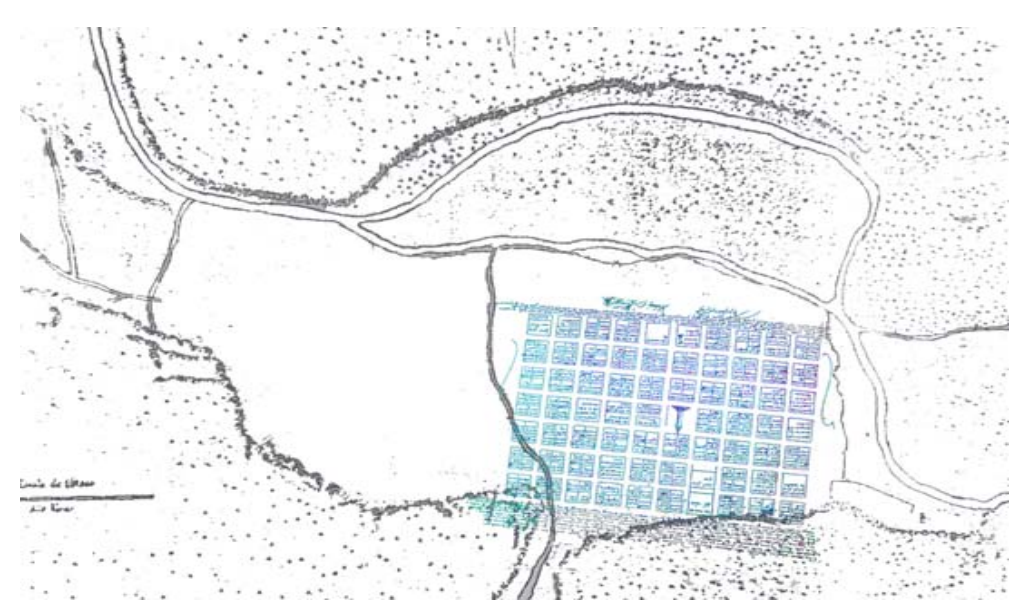

Este trazado, llamado clásico por Jorge E. Hardoy, lleva implícita la idea de centralidad, que queda reflejada en varios aspectos. ${ }^{5}$ El primero, la forma en que se diseña la ciudad, partiendo de un hipotético centro que sirve de referencia para la delineación y trazado de las calles y que luego ocupará la Plaza Mayor. Así, el único espacio público de la ciudad colonial se ubica en el mismo centro. Simultáneamente se establece cuáles son los solares que deben alojar la principal sede civil y religiosa de la sociedad colonial: el cabildo y la catedral, y se hace disponiéndolas conjuntamente frente a la Plaza Mayor; reforzando así, por segunda vez, la idea de centralidad. Asimismo, desde el primer momento que se destinan los solares, los más próximos al área central son adjudicados a las familias más importantes, con lo que se asegura su construcción, consolidando nuevamente el centro.

Si bien el proyecto de ciudad se construye con el tiempo, desde el primer momento se especifica claramente el espacio público y el privado, y sus dimensiones (calles, plazas, manzanas y solares). Estas relaciones bidimensionales se mantienen prácticamente invariables hasta el día de hoy, y constituyen las primeras huellas heredadas de la ciudad actual. Se conforma
Impresión del modelo geométrico artificial sobre el territorio natural

Montaje del modelo teórico geométrico para la ciudad de Córdoba sobre el territorio natural. El origen, la plaza se asienta sobre una topografía llana y desde allí se procede al trazado; lo cual lleva muchas veces (como es el caso) a encontrarse con accidentes topográficos que el modelo no resuelve.

Fuente: elaboración propia. Superposición a partir del plano de Lorenzo Suarez de Figueroa de 1577 y el plano de "Labergue" de 1860 , donde se grafía la hidrografía y la topografía. Fuente de los planos utilizados: Archivo Histórico Municipal de la Ciudad de Córdoba.

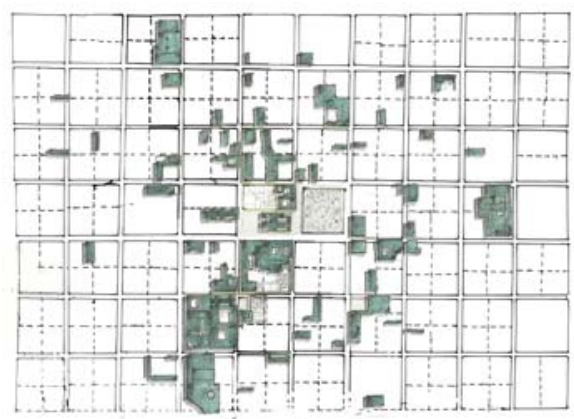

Las huellas heredadas

La traza y sus relaciones bi-dimiensionales y como un tablero de juego y los edificios patrimoniales como fichas invariables para el juego en tres dimensiones

Fuente: elaboración propia sobre plano actual de la Ciudad de Córdoba

${ }^{5}$ HARDOY, Jorge E. (1968) en HARDOY, J y TOBAR, C; editores (1968). 
así un tablero de juego que, como se ha comprobado con el tiempo, admite reglas variables en tres dimensiones, donde solo son invariables algunos edificios patrimoniales conservados.

\section{ADAPTACIÓN. Las variaciones morfológicas del modelo}

El ideario de conquista que se desea construir a base de fundar ciudades que reproducen un mismo modelo solo puede alcanzarse en parte. La geometría perfecta impuesta solo se construye parcialmente, cosa que sucederá en la mayoría de las ciudades, ya que el modelo teórico debe sufrir variaciones: primero para responder a requerimientos funcionales de cada ciudad; segundo, para adaptase a la realidad del territorio natural $y$, finalmente, porque se introducen variaciones y se superponen proyectos a medida que se va construyendo, ya sea en los primeros años o más adelante. Aparecen así los rasgos que hacen única a cada ciudad.

Las primeras variaciones que sufre el modelo en el caso de Córdoba son las adaptaciones a los requerimientos funcionales específicos, que se producen en el momento mismo de trazar el primer plano. Se dan en dos casos: el primero, la subdivisión de una manzana regular en dos más estrechas por medio de un pasaje que separa el Cabildo y la Iglesia Mayor. Por otro lado, se agrupan dos manzanas, que alojan el Convento de San Francisco, y que dejan una plazoleta en uno de sus extremos.

La segunda variación morfológica está relacionada con la geografía del lugar, que es ignorada en un primer momento, pero que con el tiempo termina por imponer sus reglas. Es así que cuando las setenta manzanas de la fundación comienzan a construirse, las que se ubican próximas al cauce del arroyo, hoy conocido como La Cañada, deben variar su geometría para adaptarse a su borde. Estos lugares de difícil construcción se van completando con lentitud y los continuos desbordes que sufre el arroyo hacen que la ocupación sufra

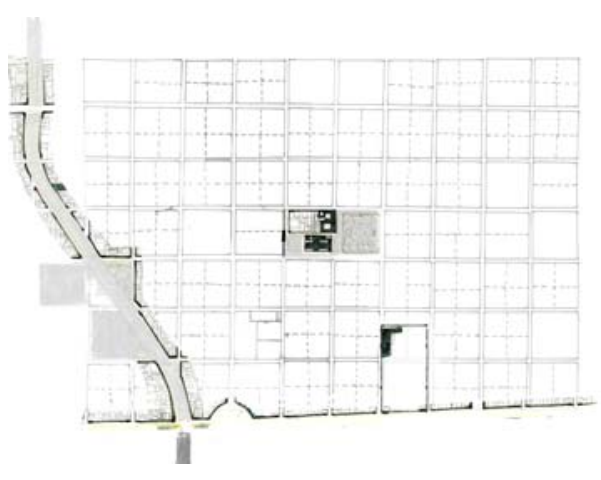

Las variaciones funcionales y topográficas Se rompe la regla de las manzanas cuadradas Fuente: elaboración propia

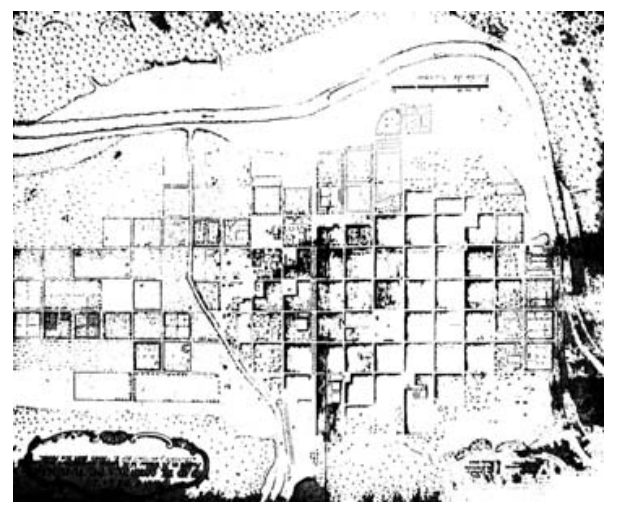

La calle ancha y el desplazamiento de la idea de centralidad

Plano de Jacinto Díaz de la Fuente. 1790

Fuente: Archivo Municipal de Córdoba 
innumerables mutaciones. Se genera un ámbito donde el tipo de manzana no es el previsto en la planta fundacional.

Pero no solo la geometría y el trazado varían, también el orden central del modelo. A pesar de que el proceso de crecimiento en las décadas inmediatamente posteriores a la fundación afirma la tendencia a la centralidad, ${ }^{6}$ esta se va trastocando a medida que la ciudad continua creciendo, ya que no lo hace de forma homogénea. Asimismo aparecen otros puntos significativos que actúan como "nuevos centros", atrayendo la ocupación hacia ellos, como es el caso de la Compañía de Jesús y el convento de San Francisco. Otro ejemplo es la apertura de la calle ancha y su alameda en 1592, que volcada hacia el oeste disecciona el modelo de Norte a Sur ${ }^{7}$. Con su ensanchamiento se produce la primera operación intencionada de variación del trazado original.
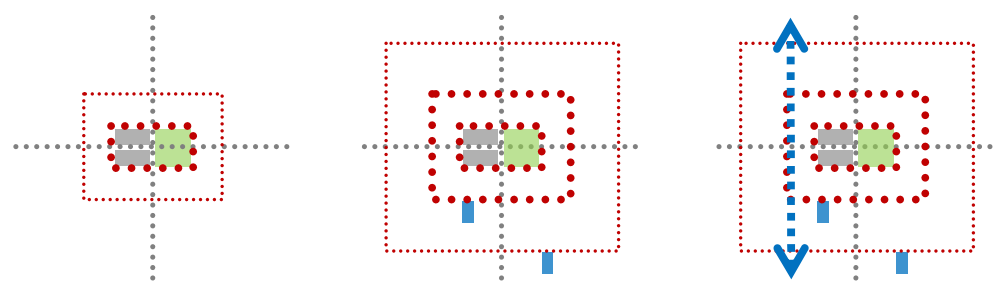

Variación de la idea de centralidad

Fuente: elaboración propia

A esta primera operación le suceden otras, décadas después,. A principios del siglo $X X$ las ciudades ya han comenzado su proceso de modernización, y es como parte de este proceso que se realizan, entre 1910 y 1930, dos operaciones de ensanchamiento dentro de los límites del área central. La primera, en sentido Este-Oeste, prácticamente en el límite norte de las setenta

\footnotetext{
${ }^{6}$ La zona central se densifica por subdivisión de los solares originales antes incluso, que se ocupe la totalidad de las setenta manzanas. Simultáneamente comienzan a aparecer los primeros "comercios", que contribuyen a acentuar su centralidad.

${ }_{7}^{7}$ Actual calle Vélez Sarsfield, una de las avenidas principales del área central de la ciudad
} 
manzanas; y la segunda en sentido Norte-Sur en el lado Oeste. ${ }^{8}$ Éstas terminan por dividir el trazado original en tres sectores diferenciados que crecen en las décadas posteriores con distinta dinámica y que alteran el modelo original superponiendo una jerarquía viaria a un trazado originalmente indiferenciado.

\section{Dos espacios identitarios}

Podríamos reconocer a partir de cómo ha variado y se ha adaptado el modelo original y de su representación en un plano, aquellos espacios que definen la identidad del área central de la ciudad de Córdoba. Nos centraremos en los dos más significativos, los que permanecen en el imaginario de todo aquel que vive en la ciudad o la visita. Nos referimos al arroyo de La Cañada y al entorno de la plaza San Martín.

\section{La Cañada}

El actual espacio conocido como La Cañada tiene su origen en un pequeño cauce de arroyo un tanto indefinido que tiene su final en el Río Suquía.

El primer plano para la ciudad desconoce por completo su existencia, y la dificultad por asentarse en una zona inundable provoca que, durante mucho tiempo no se consolide. Sus constantes desbordes hacen que sus márgenes sean ocupados una y otra vez. En un primer momento con asentamientos precarios que desaparecen tras cada inundación. Sin embargo una regla parece clara desde el inicio, la geometría no puede con la naturaleza, obligando a variar y combinar aleatoriamente el damero con el orden irregular de su cauce.

Dos planos de fines del siglo XIX muestran detalladamente la forma de ocupación de este sector antes de su consolidación. ${ }^{9}$ Es la última inundación

\footnotetext{
${ }^{8}$ La primera corresponde a la actual Avenida Colón y la segunda a la Avenida Maipú

${ }^{9}$ Los planos a los que nos referimos son: el de J. E. Sturtz de 1889, reproducido en BARBIERI, S. y BOIXADÓS, C. (2005) y el Catastro Machado de 1890.
}
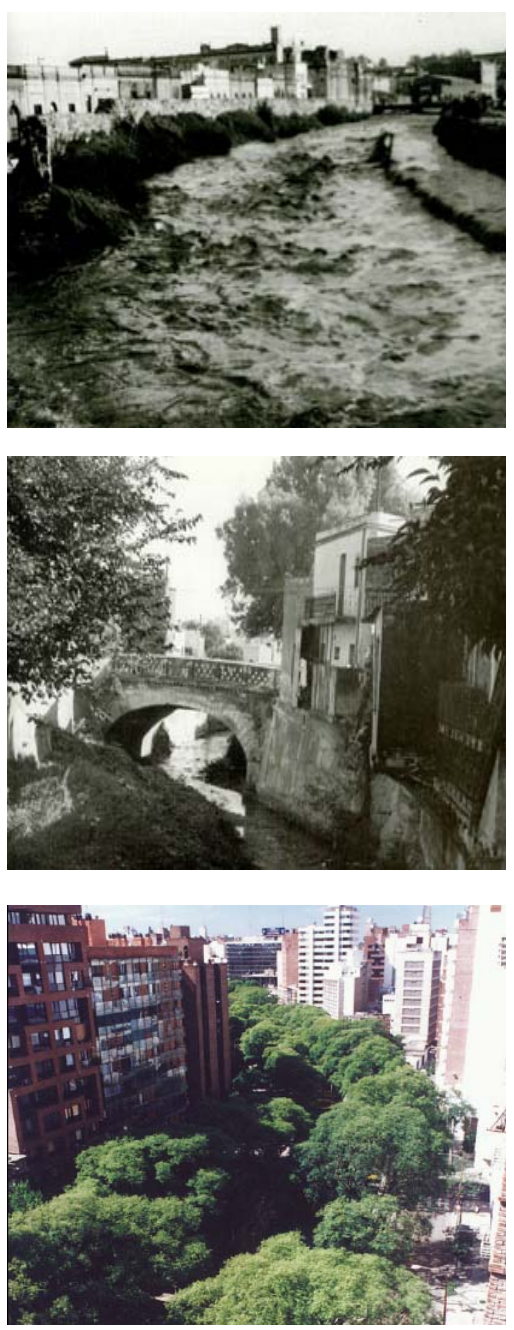

La identidad de la Cañada

Diferentes momentos de su transformación Fuente: BARBIERI, S. y BOIXADÓS, C. (2005) y PeCBA 


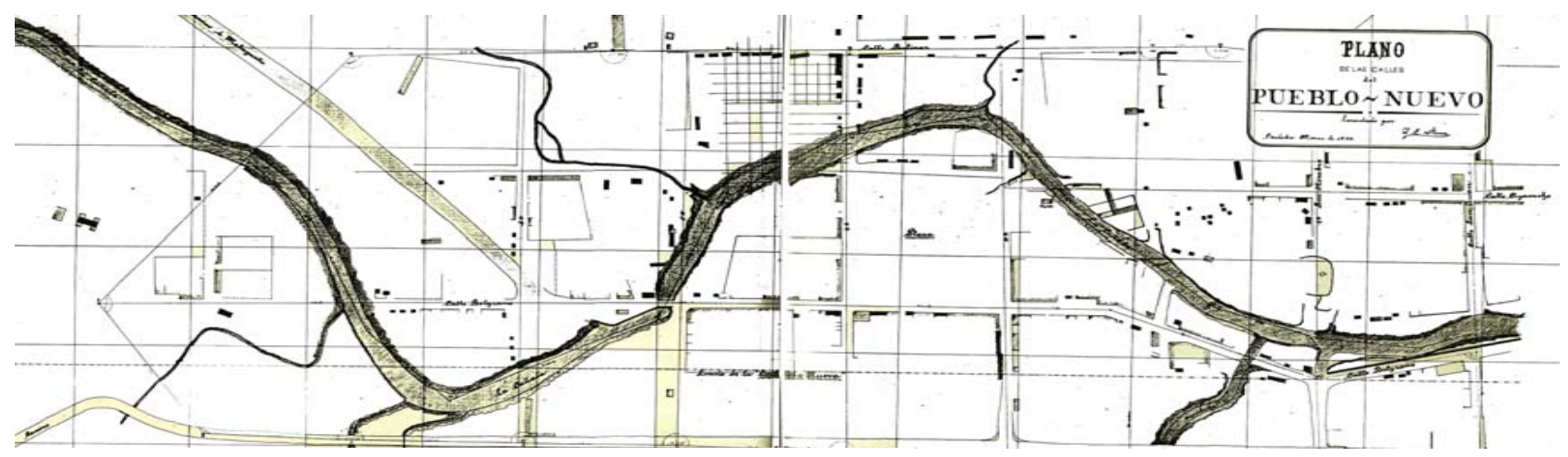

Los primeros asentamientos sobre la Cañada. Plano de J. E. Stutz de 1889 Fuente: BARBIERI, S y BOIXADÓS, C (2005)

en el año 1939 la que obliga a pensar en su canalización definitiva, una gran obra de infraestructura, cuyo proceso comienza en 1942 y concluye en $1957 .{ }^{10}$ A partir de esta fecha queda definido el trazado, lo que origina manzanas de planta triangular. Asimismo empieza a consolidarse el paisaje dominado por el calicanto y el gran porte de su vegetación (tipas) que conocemos actualmente y que son símbolo de la imagen de Córdoba.

Si el trazado definitivo del centro histórico tarda cuatro siglos en consolidarse, La Cañada es un proyecto que se construye con el tiempo, a partir de acciones individuales y de las diferentes normas que se van superponiendo.

\section{Entorno de la Plaza San Martín}

Definida desde el momento de la fundación como nodo central de la ciudad, el entono de la plaza San Martín es seguramente el espacio donde más ideas y proyectos se han superpuesto a lo largo de su historia. Es el lugar central y donde se ubicaron los equipamientos más representativos. Varios son los edificios que aún hoy dan cuenta de aquella época. Marina Waisman dice al respecto que ninguna ciudad argentina posee una arquitectura semejante

\footnotetext{
${ }^{10}$ GOYTIA, Noemí (1995). en GOYTIA, N y FOGLIA, M.E.(1995).
} 
concentrada en tan pocas manzanas como Córdoba. ${ }^{11}$ De ahí que este espacio haya sido un lugar de especial interés, donde se han ensayado diferentes normas que han intentado preservar el patrimonio pero también reglar la nueva arquitectura.

En 1950, La Padula, arquitecto italiano radicado en Córdoba, procede al relevamiento del patrimonio histórico de las manzanas próximas a la actual plaza San Martín y concluye con una ordenanza en $1954 .{ }^{12}$ Su ámbito de aplicación se limita a las fachadas que rodean la plaza, para las cuales establece las alturas y alineaciones que deben tener las nuevas edificaciones para que estén en relación con los edificios históricos próximos. De esta forma se define el perfil que debe delimitar la plaza.

Años más tarde, bajo la dirección de otro arquitecto italiano, Enrico Tedeschi, se redacta una nueva ordenanza para normar "un ámbito con gran presencia de monumentos históricos y religiosos." ${ }^{13}$ Con esta ordenanza se define la volumetría de las manzanas en función de los edificios patrimoniales que son considerados como "hitos que deben estar rodeados por ámbitos adecuados a su escala e importancia artística, y conexos entre sí por una red de espacios que permitan continuidad de recorridos y de visuales, aprovechando la presencia de los monumentos desde los puntos de vista más numerosos y variados posibles". ${ }^{14}$ El ámbito de aplicación abarca unas veinte manzanas. Su aplicación modifica la identidad del sector proponiendo una volumetría diferente al resto del centro histórico, cambiando algunas alineaciones, proponiendo alturas obligatorias, ${ }^{15}$ pero también superponiendo una trama de recorridos peatonales que jerarquizan y vinculan los edificios históricos.

\footnotetext{
${ }^{11}$ WAISMAN, Marina (1970) "Córdoba y su arquitectura" en Revista Summa n 30, 1970. págs. 26-69. Argentina.

${ }^{12}$ Ordenanza N ${ }^{\circ} 4273$ del 30 de julio de 1954

${ }_{14}^{13}$ Decreto Ordenanza $172 / 67$

${ }^{14}$ Decreto Ordenanza 172/67

${ }^{15}$ Hasta entonces siempre se habían definido alturas máximas permitidas
}

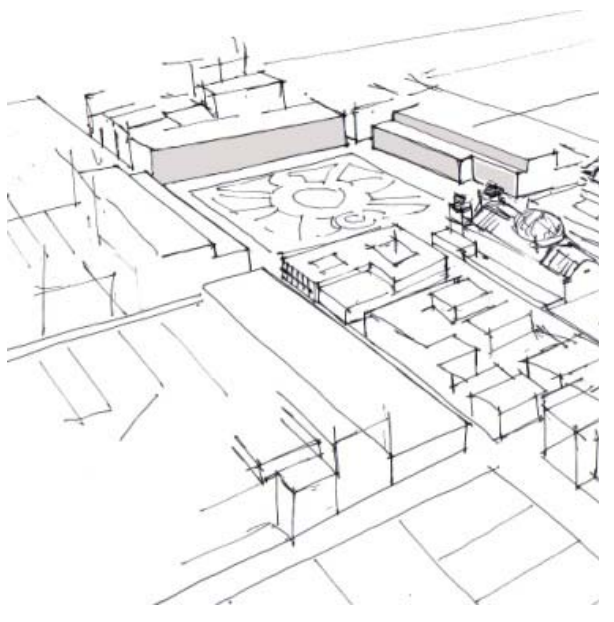

Ordenanza de La Padila Fuente: elaboración propia

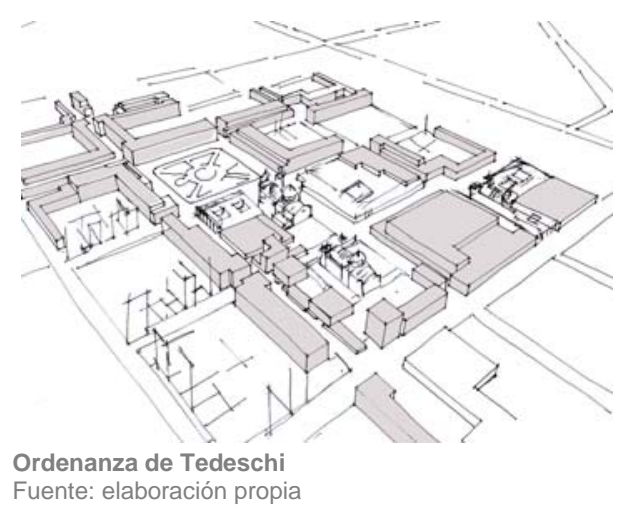


Finalmente en 1985 se elabora la ordenanza de Ocupación del Suelo y preservación de Ámbitos Históricos en el Área Central. Ésta considera como área central a un sector mucho más amplio, que supera incluso las 70 manzanas de la fundación. Es en este sector donde la norma general hace una excepción y trabaja manzana a manzana para definir su volumetría de acuerdo con los edificios patrimoniales e intentando garantizar las visuales hacia ellos, algo similar a lo que hacía Tedeschi.

El modelo y las construcciones de origen, sumadas a la superposición de estas tres ordenanzas, termina por definir la imagen final de este sector, que lo hace reconocible frente a otros.

\section{DIALÉCTICA ENTRE EL MODELO Y SUS VARIACIONES}

No se puede negar la importancia del damero en la configuración de las ciudades latinoamericanas de origen español. Este trazado ha marcado, en parte, la identidad del área central de nuestras ciudades. La carga simbólica y su impronta formal son tan potentes que permanecen hasta el día de hoy. El modelo geométrico ha influido, de una u otra manera, en la construcción de la ciudad a lo largo de casi toda su historia. Si bien éste se utiliza repetidamente, en ninguno de los ejemplos se repite el original, sino que presenta rasgos y variaciones propias. Uno de los casos más claros es el de los nuevos barrios que se generan a finales del siglo XIX en Córdoba, los conocidos como barrios pueblos. Lo interesante de esta primera expansión es que, pasados casi trescientos años desde la fundación, es nuevamente el modelo en damero el que sirve para ordenar estos barrios, aunque variando sus proporciones y superponiendo otras ideas como las higienistas o las de ornato público.

La herencia de la cuadrícula, aunque en alguna época denostada como instrumento de diseño de ciudades, ha demostrado trascender en el tiempo y ser una constante en la evolución de la ciudad de Córdoba. Y, aunque no

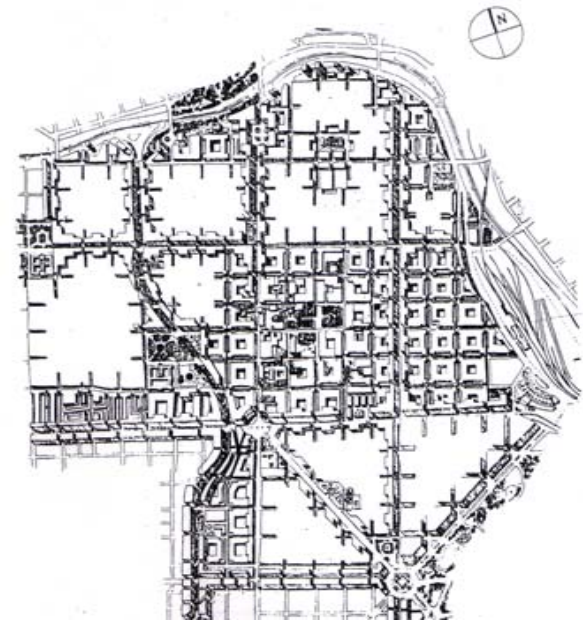

Ordenanzas de 1985

Fuente: Ordenanza de Ocupación del suelo y Preservación de Ámbitos Históricos. 
podamos negar el trazado impuesto como huella imborrable de identidad, también las variaciones, adaptaciones y posteriores reflexiones son tan singulares y únicas como él mismo. El modelo geométrico se superpone y adapta a las preexistencias y es justamente esta sinergia lo que le confiere su identidad.

En un primer estadio, éste es genérico y solo admite variantes dimensionales. ${ }^{16}$ No obstante no puede deshacerse de la disciplina que le impone el medio natural, ni de los requerimientos específicos de cada grupo fundador. Así aparece la traza de La Cañada y los hitos de las manzanas de la CatedralCabildo, y el Convento de San Francisco. También la construcción en el tiempo juega un papel relevante en la configuración de los rasgos singulares. El área central, como lugar representativo, es objeto de continuas reflexiones. En este caso, éstas pasan por conjugar la nueva arquitectura con los edificios históricos y terminan por moldear su identidad. Es así que el modelo de retícula no jerarquizada, donde solo destaca la plaza central, varía de forma considerable a partir de operaciones intencionadas, pero también de otras que lo son menos. Es este proceso de adaptación, que dura décadas, el que termina por configurar una realidad muy diferente a aquel modelo que le da origen, pero que sin embargo, guarda algunas marcas originales que permanecen hasta el día de hoy y que contribuyen a conformar su identidad.

Frente a la universalidad que se supone a los modelos en damero heredados de la fundación hispana, se contrapone la realidad de su construcción en el tiempo. Es en este proceso que cada ciudad va construyendo los elementos de su propia identidad. La suma de la geometría de origen, la combinación de diferentes modificaciones y los proyectos y reflexiones que se producen sobre el modelo original crean los espacios que la identifican y aquellos que la hacen única frente a otras ciudades con idéntico patrón.

\footnotetext{
${ }^{16}$ Entre una ciudad y otra el modelo admitió algunas variantes como el ancho de las calles o las dimensiones de las manzanas.
}

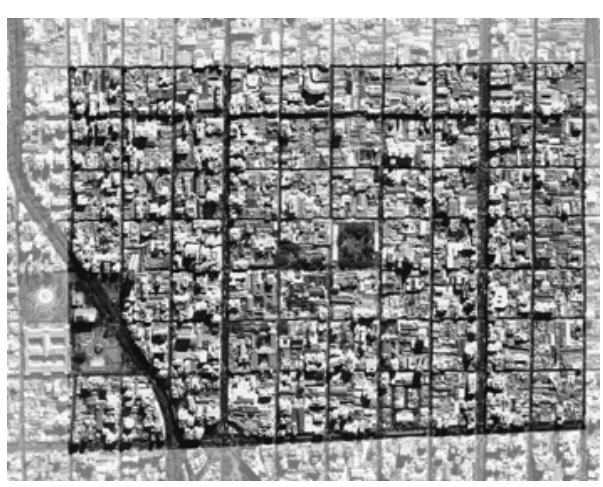

El área central hoy

Fuente: Municipalidad de Córdoba 


\section{BIBLIOGRAFÍA}

AAVV. (1994) (FOGLIA, GOYTIA, GIORDANO, FREGUGLIA, MARTINEZ, GAMBONE, CAMISA, MALIK, VENTURINI, ORTEGA, FRANCHELLO, DEMARCO, BOIXADOS). La cuadrícula en el desarrollo de la ciudad Hispanoamericana. El Caso de Córdoba. Tomos I y II. Departamento de publicaciones de la Facultad de Arquitectura, Urbanismo y Diseño de la Universidad Nacional de Córdoba. Córdoba

HARDOY, Jorge E. (1968) Dos mil años de urbanización en América Latina en HARDOY, J y TOBAR, C; editores (1968). El proceso de urbanización en América Latina

BARBIERI, Sergio y BOIXADÓS, Cristina (2005) El cauce viejo de La Cañada. Fotografías 1885-1945. Editado por Fundación Banco de Alimentos de Córdoba

GOYTIA, Noemí (1995). "La infraestructura y el equipamiento como signo de progreso en la ciudad Americana: el caso de Córdoba-Argentina" en GOYTIA, Noemí y FOGLIA, María Elena. (1995). Los procesos de Modernización en Córdoba. Departamento de publicaciones de Facultad de Arquitectura, Urbanismo y Diseño de la Universidad Nacional de Córdoba. Córdoba

AAVV. (1997) La ciudad Hispanoamericana. El sueño de un orden. Publicado por el Centro de Estudios Históricos de Obras Públicas y Urbanismo (CEHOPU), el Centro de Estudios y Experimentación de Obras Públicas y el Ministerio de Fomento de España. Edición original de 1989 con motivo de la exposición del mismo título presentada en Madrid.

PECBA. Plan Estratégico de la ciudad de Córdoba. Publicación de 2005. Municipalidad de Córdoba.

Ordenanza Nº4273 del 30 de julio de 1954. Archivo Municipal de Córdoba

Decreto Ordenanza 172/67. Archivo Municipal de Córdoba

Este trabajo fue realizado con el soporte del Comisionado para la Universidad e Investigación del

Departamento de Innovación, Universidades y Empresas de la Generalitat de Cataluña y del Fondo Social Europeo. 